\title{
INVARIANT METHODS IN CLASSICAL DIFFERENTIAL GEOMETRY*
}

\author{
BY W. C. GRAUSTEIN
}

There are two well known developments of classical differential geometry by invariant methods which are systematic and comprehensive, namely those of Cesàro $\dagger$ and Ricci. $\ddagger$ Ricci's tensor analysis is so familiar as to need no detailed description, and the methods of Cesàro may perhaps best be set forth by comparing them with those of Ricci. In making this comparison there are two questions of major importance to consider, the one having to do with the point of departure of the theory, and the other with the method of differentiation.

Ricci based his developments on an analytic representation of the manifold under consideration. On the other hand, it was a matter of principle with Cesàro to get along without a fixed coordinate system to which to refer the manifold. Perhaps it is this strict adhesion to purely intrinsic geometry that has stood in the way of a more general adoption of Cesàro's methods. In any case, the opinion seems to be generally held that a more powerful theory is obtainable by combining the purely intrinsic methods with invariant methods based on representations by means of parameters or coordinates.

In contrast to Ricci's method of covariant differentiation, Cesàro employs the older method of intrinsic differentiation, differentiation along a curve with respect to the arc of the curve. $\$$ It is probably the general opinion that here, too, Ricci's choice is the better one. This opinion is undoubtedly justifiable in the case of manifolds of higher dimensionality, but it is a question whether it can be upheld in the case of the manifolds of classical differential geometry.

* An address presented to the Society at the request of the program committee, April 18, 1930.

$\dagger$ Lezioni di Geometria Intrinseca, 1896.

$\ddagger$ Lezioni sulla Teoria delle Superficie, 1898; also, Ricci and Levi-Civita, Méthodes de calcul différentiel et absolu et leurs applications, Mathematische Annalen, vol. 54 (1901), pp. 125-201.

$\S$ The method was used earlier by various writers, notably, Lamé, Bonnet, Gilbert, and Enneper. 
In comparing the two methods in this case, let us, for example, consider the theory of a surface in euclidean 3-space. Fundamental in this theory are the three quadratic differential forms of the surface, and linear differential forms which, when equated to zero, represent families of curves on the surface. From the point of view of invariant theory, the important problem consists in finding the invariants of this system with respect to changes of parameters. We pass over the algebraic invariants of the system,- they are readily found. It is invariants depending on further differentiation which interest us.

Intrinsic differentiation of invariants yields new invariants as a result. On the other hand, covariant differentiation does not give rise immediately to new invariants. Applied to invariants or to tensors, it yields first new tensors, and only secondarily, by application of algebraic processes to these tensors, does it give rise to new invariants. It is in this way, for example, that geodesic curvature is introduced.

Equations expressed in terms of the components of tensors and their covariant derivatives are not, in general, invariant. This is the case, for example, for the equations corresponding to those of Codazzi. As a matter of fact, Ricci does not leave final results, such as these, in tensor form, but reverts to the use of actual invariants and their intrinsic derivatives.

But the intrinsic derivatives are, in themselves, sufficient. Covariant differentiation is only an intermediate process which is not only unnecessary, but also disadvantageous in that, in dealing essentially with tensors rather than with invariants, it requires the use of non-invariant quantities and equations.

We have noted that Cesàro's use of intrinsic differentiation was divorced from any fixed analytic representation of the surface. The introduction of intrinsic differentiation in connection with a parametric representation of the surface has been carried out by various authors, notably, Lilienthal, ${ }^{*}$ Knoblauch, $\dagger$ and Blaschke. $\ddagger$ However, in none of these treatments

* Über geodätische Krümmung, Mathematische Annalen, vol. 42 (1893), pp. 511-516; Vorlesungen über Differentialgeometrie, vol. II, 1913, Part IV.

$\dagger$ Grundlagen der Differentialgeometrie, 1913, pp. 56, 89, 191, 201, 205, 255, $351,389,522,572$.

$\ddagger$ Vorlesungen über Differentialgeometrie, vol. I, 3d edition, 1930, pp. $123-145,181-190,289-292$. 
is full use made of the connection with the parametric representation. In fact, the importance of this connection for the development of a comprehensive and powerful theory seems not to have been completely realized.

The theory set forth in this paper aims to emphasize this connection, as well as to show the advantages of the use of directional derivatives. It also introduces two further tools, Jacobians and a modified form of intrinsic differentiation. Jacobians are employed in establishing the connection with the parametric representation, in obtaining invariant vectors and functions, and in introducing directional derivatives. The modified directional derivatives cooperate with the ordinary directional derivatives to yield a simpler, and, it is hoped, a more powerful, theory.*

The application of the theory has been restricted here to surfaces in euclidean 3 -space. It will be evident, however, that the methods employed have a wider field of usefulness.

\section{A. The Use of Jacobians}

1. Invariant Vectors. Let $x=x(u, v)$ :

$$
x_{1}=x_{1}(u, v), \quad x_{2}=x_{2}(u, v), \quad x_{3}=x_{3}(u, v) \dagger
$$

be a real surface, $S$, referred to a system of real curves, and let

$$
u=u\left(u^{\prime}, v^{\prime}\right), v=v\left(u^{\prime}, v^{\prime}\right)
$$

be an arbitrary change of parameters, with Jacobian $\Delta \neq 0$.

Let $\phi(u, v)$ and $\psi(u, v)$ be two functions of $u$ and $v$, and denote their Jacobian by $[\phi, \psi]$ :

$$
[\phi, \psi]=\phi_{u} \psi_{v}-\phi_{v} \psi_{u} .
$$

If $\phi$ and $\psi$ are transformed by (1) into $\phi^{\prime}$ and $\psi^{\prime}$ so that $\phi^{\prime} \equiv \phi$ and $\psi^{\prime} \equiv \psi$, then

$$
\left[\phi^{\prime}, \psi^{\prime}\right]=\Delta[\phi, \psi]
$$

* The theory, as set forth, is a revision of a recent paper by the author, Méthodes invariantes dans la géométrie infinitésimale des surfaces, Mémoires de l'Académie Royale de Belgique (Classe des Sciences), (2), vol. 11 (1929). The reader is referred to this paper for references concerning the details, and also for examples illustrating the applications of the theory.

$\dagger$ It is assumed that all functions are real, single-valued, and analytic throughout a certain domain of the real variables $u, v$. 
Thus, the Jacobian of two absolutely invariant functions is a relative invariant of weight unity.

A vector whose components are all relative invariants of the same weight is said to be a relatively invariant vector.

If the components of a vector are the Jacobians of functions which are absolutely invariant with respect to an arbitrary change of parameters, the vector is relatively invariant.

A great majority of the important relatively invariant vectors have components formed essentially in this fashion.

2. Invariant Vectors Normal to the Surface. The familiar vector*

$$
\alpha=\overbrace{x_{u} x_{v}}
$$

is a relatively invariant vector of weight one:

$$
\alpha^{\prime}=\Delta \alpha .
$$

It gives rise to the unit vector, $\zeta$, normal to the surface:

Since

$$
\zeta=\frac{\alpha}{(\alpha \mid \alpha)^{1 / 2}}
$$

$$
\begin{aligned}
\left(\alpha^{\prime} \mid \alpha^{\prime}\right)^{1 / 2} & = \pm \Delta(\alpha \mid \alpha)^{1 / 2} \\
\zeta^{\prime} & = \pm \zeta
\end{aligned}
$$

where the plus sign or the minus sign is to be taken according as $\Delta>0$ or $\Delta<0$. Hence $\zeta$ is an absolutely invariant vector, except perhaps for a change of signs.

From $x$ and $\zeta$ may be formed the two further vectors,

$$
\gamma=\widetilde{\zeta_{u} \zeta_{v}}, \quad \beta=-\overbrace{x_{u} \zeta_{v}}-\overbrace{\zeta_{u} x_{v}}
$$

normal to the surface.

The components of the three vectors $\alpha, \beta, \gamma$ may all be expressed in terms of Jacobians of invariant functions. We have:

$$
\begin{aligned}
& \alpha_{1}=\left[x_{2}, x_{3}\right], \quad \beta_{1}=-\left[x_{2}, \zeta_{3}\right]-\left[\zeta_{2}, x_{3}\right], \quad \gamma_{1}=\left[\zeta_{2}, \zeta_{3}\right], \\
& \left\{\alpha_{2}=\left[x_{3}, x_{1}\right], \quad \beta_{2}=-\left[x_{3}, \zeta_{1}\right]-\left[\zeta_{3}, x_{1}\right], \quad \gamma_{2}=\left[\zeta_{3}, \zeta_{1}\right],\right. \\
& \alpha_{3}=\left[x_{1}, x_{2}\right], \quad \beta_{3}=-\left[x_{1}, \zeta_{2}\right]-\left[\zeta_{1}, x_{2}\right], \quad \gamma_{3}=\left[\zeta_{1}, \zeta_{2}\right] .
\end{aligned}
$$

* Vector notation. If $a: a_{1}, a_{2}, a_{3}$, and $b: b_{1}, b_{2}, b_{3}$ are ordered triples of numbers, their inner and outer products shall be denoted by $(a \mid b)$ and $\widetilde{a b}$ respectively:

$$
\widehat{a b:} \quad \begin{gathered}
(a \mid b)=a_{1} b_{1}+a_{2} b_{2}+a_{3} b_{3} \\
a_{2} b_{3}-a_{3} b_{2}, a_{3} b_{1}-a_{1} b_{3}, a_{1} b_{2}-a_{2} b_{1} .
\end{gathered}
$$


Hence, $\alpha, \beta, \gamma$ are relatively invariant vectors of weight one, except that, in the case of $\beta$, the signs may change.

The vectors $\alpha, \beta, \gamma$ stand in close relationship, respectively, to the three fundamental differential forms of $S$. We write these forms, with their discriminants, as follows:

$$
\begin{aligned}
& A=(d x \mid d x)=E d u^{2}+2 F d u d v+G d v^{2}, \quad D^{2}=E G-F^{2}, \\
& B=-(d x \mid d \zeta)=e d u^{2}+2 f d u d v+g d v^{2}, \quad d^{2}=e g-f^{2}, \\
& C=(d \zeta \mid d \zeta)=\mathcal{E} d u^{2}+2 \mathcal{F} d u d v+G d v^{2}, \quad \mathcal{D}^{2}=\mathcal{E} G-\mathcal{F}^{2} \text {. }
\end{aligned}
$$

The inner product of two of the vectors $\alpha, \beta, \gamma$, or of one of these vectors with itself, is relatively invariant with respect to a change of parameters, and absolutely invariant with respect to rigid motions. The six invariants obtained in this way are essentially the fundamental algebraic invariants of the three quadratic forms with respect to changes of parameters:

\section{Form Discriminant Simultaneous Invariant}

$$
\begin{array}{lll}
A & D^{2}=(\alpha \mid \alpha), & I(A, B)=(\alpha \mid \beta), \\
B & D^{2}=(\alpha \mid \gamma), & I(A, C)=(\beta \mid \beta)-2(\alpha \mid \gamma), \\
C & D^{2}=(\gamma \mid \gamma), & I(B, C)=(\beta \mid \gamma) .
\end{array}
$$

Since $\alpha, \beta, \gamma$ are all normal to $S$, and $\alpha$ is never a null vector, $\gamma$ and $\beta$ are scalar multiples of $\alpha$. The multipliers are respectively $K$ and $K^{\prime}$, the total and mean curvatures of the surface:

$$
\gamma=K \alpha, \quad \beta=K^{\prime} \alpha .
$$

When these values of $\gamma$ and $\beta$ are substituted into (2), the six invariants in (2) become functions of $(\alpha \mid \alpha), K, K^{\prime}$. Hence, all the algebraic relative invariants of the three fundamental forms with respect to changes of parameters are functions of $(\alpha \mid \alpha), K, K^{\prime}$. But $(\alpha \mid \alpha), K, K^{\prime}$ form a complete system of absolute invariants, with respect to rigid motions, of the three vectors $\alpha, \beta, \gamma$. Hence, the system of absolute invariants with respect to rigid motions of these three vectors, which are themselves relatively invariant with respect to changes of parameters, is identical with the system of algebraic relative invariants of the three fundamental forms with respect to changes of parameters. 
3. Invariant Vectors Tangent to the Surface. If $\phi(u, v)$ is a function which is absolutely invariant with respect to a change of parameters, there is associated with it the relatively invariant vector

$$
[x, \phi]: \quad\left[x_{1}, \phi\right],\left[x_{2}, \phi\right],\left[x_{3}, \phi\right],
$$

and also the vector

$$
\Phi=\frac{1}{D}[x, \phi]=\frac{1}{D}\left(\phi_{v} x_{u}-\phi_{u} x_{v}\right),
$$

which is absolutely invariant except perhaps for a change of signs.

If $\phi$ is not a constant, the equation $\phi(u, v)=$ const. represents on $S$ a family of curves, and the vector $\Phi$ at a point $P$ of $S$ lies on the tangent, at $P$, to the curve of the family which passes through $P$. We agree to direct the curve so that the positive direction is that of $\Phi$.

Suppose that $\psi(u, v)=$ const. represents a second family of curves on $S$ and let

$$
\Psi=\frac{1}{D}[x, \psi]
$$

be the corresponding absolutely invariant vector.

The inner product of one of the vectors $\Phi, \Psi$ with itself, or with the other, is evidently an absolute invariant. The invariants thus obtained are simply the familiar differential parameters of the first order:

$$
\Delta_{1} \phi=(\Phi \mid \Phi), \quad \Delta_{1}(\phi, \psi)=(\Phi \mid \Psi) .
$$

We have, also,

where

$$
\Theta_{1}^{2}(\phi, \psi)=(\widetilde{\Phi \Psi} \mid \widetilde{\Phi \Psi})
$$

$$
\Theta_{1}(\phi, \psi)=\frac{1}{D}[\phi, \psi]
$$

The usual formulas for the angle $\omega$ from the directed curve $\phi=$ const. to the directed curve $\psi=$ const., namely,

$$
\cos \omega=\frac{\Delta_{1}(\phi, \psi)}{\left(\Delta_{1} \phi\right)^{1 / 2}\left(\Delta_{1} \psi\right)^{1 / 2}}, \quad \sin \omega=\frac{\Theta_{1}(\phi, \psi)}{\left(\Delta_{1} \phi\right)^{1 / 2}\left(\Delta_{1} \psi\right)^{1 / 2}},
$$


now reduce to the obvious forms

$$
\cos \omega=\frac{(\Phi \mid \Phi)}{(\Phi \mid \Phi)^{1 / 2}(\Psi \mid \Psi)^{1 / 2}}, \quad \sin \omega=\frac{\Theta_{1}(\phi, \psi)}{(\Phi \mid \Phi)^{1 / 2}(\Psi \mid \Psi)^{1 / 2}} .
$$

Since the length of the vector $\Phi$ is $\left(\Delta_{1} \phi\right)^{1 / 2}$, the vector $\zeta \Phi$ gives, in direction and length, the directional derivative of $\phi(u, v)$ of maximum value. It is the generalization of the gradient of a function in the plane.

The unit vector, $\partial x / \partial s$, in the direction of the vector $\Phi$ is

$$
\frac{\partial x}{\partial s}=\bar{\Phi}=\frac{\Phi}{(\Phi \mid \Phi)^{1 / 2}}=\frac{1}{D} \frac{[x, \phi]}{\left(\Delta_{1} \phi\right)^{1 / 2}} .
$$

Hence, the rate of change of an arbitrary function $\chi(u, v)$ in the positive direction of the curve $\phi(u, v)=$ const. is

$$
\frac{\partial \chi}{\partial s}=\frac{1}{D} \frac{[\chi, \phi]}{\left(\Delta_{1} \phi\right)^{1 / 2}} .
$$

4. Differential Parameters of the Second Order. Geodesic Curvature. Jacobians of invariant functions may be employed to form scalar invariants as well as invariant vectors.

For example, the differential parameter $\Delta_{2} \phi$, expressed in terms of Jacobians in a form which is obviously invariant with respect to a change of parameters, is

$$
\Delta_{2} \phi=\frac{1}{D} \sum_{i=1}^{3}\left[x_{i}, \Phi_{i}\right]
$$

Equivalent to this is the expression

$$
\Delta_{2} \phi=\frac{1}{D}\left[\frac{\partial}{\partial v}\left(x_{u} \mid \Phi\right)-\frac{\partial}{\partial u}\left(x_{v} \mid \Phi\right)\right],
$$

which, when for $\Phi$ is substituted the value given by (3), expands in to the usual form.

Again, the geodesic curvature of a curve $\phi(u, v)=$ const. is given by

$$
\frac{1}{\rho}=-\frac{1}{D} \sum_{i=1}^{3}\left[x_{i}, \bar{\Phi}_{i}\right]
$$

For this relation may be put into the form 


$$
\frac{1}{\rho}=\frac{1}{D}\left[\frac{\partial}{\partial u}\left(x_{v} \mid \frac{\partial x}{\partial s}\right)-\frac{\partial}{\partial v}\left(x_{u} \mid \frac{\partial x}{\partial s}\right)\right]
$$

and may then be expanded into the classical formula of Bonnet.

\section{B. Directional Derivatives}

5. Condition of Integrability. Let there be given a system of curves on $S$ consisting of the family of curves $C: \phi(u, v)=$ const., and the family of curves $C^{\prime}: \psi(u, v)=$ const. Assume that $[\phi, \psi]>0$, that is, that the curves of the families are so directed that $0<\omega<\pi$, where $\omega$ is the angle from the directed curve $C$ to the directed curve $C^{\prime}$, measured in the positive sense.

The unit vectors, $\xi$ and $\xi^{\prime}$, in the positive directions of the curves $C$ and $C^{\prime}$, are, by (4),

$$
\begin{aligned}
\xi & =\frac{\partial x}{\partial s}=\frac{1}{D}\left[\frac{\phi_{v}}{\left(\Delta_{1} \phi\right)^{1 / 2}} x_{u}-\frac{\phi_{u}}{\left(\Delta_{1} \phi\right)^{1 / 2}} x_{v}\right] \\
\xi^{\prime} & =\frac{\partial x}{\partial s^{\prime}}=\frac{1}{D}\left[\frac{\psi_{v}}{\left(\Delta_{1} \psi\right)^{1 / 2}} x_{u}-\frac{\psi_{u}}{\left(\Delta_{1} \psi\right)^{1 / 2}} x_{v}\right] .
\end{aligned}
$$

Hence

and

$$
\begin{aligned}
& x_{u} \sin \omega=-\frac{\psi_{u}}{\left(\Delta_{1} \psi\right)^{1 / 2}} \xi+\frac{\phi_{u}}{\left(\Delta_{1} \phi\right)^{1 / 2}} \xi^{\prime}, \\
& x_{v} \sin \omega=-\frac{\psi_{v}}{\left(\Delta_{1} \psi\right)^{1 / 2}} \xi+\frac{\phi_{v}}{\left(\Delta_{1} \psi\right)^{1 / 2}} \xi^{\prime},
\end{aligned}
$$

$$
d x \sin \omega=-\frac{d \psi}{\left(\Delta_{1} \psi\right)^{1 / 2}} \xi+\frac{d \phi}{\left(\Delta_{1} \phi\right)^{1 / 2}} \xi^{\prime} .
$$

Thus, the linear element of the surface, referred to the system of curves $C$ and $C^{\prime}$, is

(9) $\sin ^{2} \omega(d x \mid d x)=\frac{d \psi^{2}}{\Delta_{1} \psi}-2 \cos \omega \frac{d \psi}{\left(\Delta_{1} \psi\right)^{1 / 2}} \frac{d \phi}{\left(\Delta_{1} \phi\right)^{1 / 2}}+\frac{d \phi^{2}}{\Delta_{1} \phi}$.

The differentials of arc, $d s$ and $d s^{\prime}$, of the curves $C$ and $C^{\prime}$, are, then, respectively,

$$
d s=-\frac{d \psi}{\sin \omega\left(\Delta_{1} \psi\right)^{1 / 2}}, \quad d s^{\prime}=+\frac{d \phi}{\sin \omega\left(\Delta_{1} \phi\right)^{1 / 2}} .
$$


The arcs are measured in the positive directions of the curves, and the signs in (10) result from the fact that the functions $\phi$ and $\psi$ increase in the directions which lie to the left of the curves $C$ and $C^{\prime}$, when these curves, looked at from the side of $S$ in the positive direction of the normal $\zeta$, are traced in their positive senses.

The directional derivatives, $\partial \chi / \partial s$ and $\partial \chi / \partial s^{\prime}$, of an arbitrary function $\chi(u, v)$ in the positive directions of the curves $C$ and $C^{\prime}$, are, by (5),

$$
\begin{aligned}
& \frac{\partial \chi}{\partial s}=\frac{1}{D}\left[\frac{\phi_{v}}{\left(\Delta_{1} \phi\right)^{1 / 2}} \chi_{u}-\frac{\phi_{u}}{\left(\Delta_{1} \phi\right)^{1 / 2}} \chi_{v}\right], \\
& \frac{\partial \chi}{\partial s^{\prime}}=\frac{1}{D}\left[\frac{\psi_{v}}{\left(\Delta_{1} \psi\right)^{1 / 2}} \chi_{u}-\frac{\psi_{u}}{\left(\Delta_{1} \psi\right)^{1 / 2}} \chi_{v}\right] .
\end{aligned}
$$

Solving these equations for $\chi_{u}$ and $\chi_{v}$, we have

$$
\begin{aligned}
& \chi_{u}=-\frac{\psi_{u}}{\sin \omega\left(\Delta_{1} \psi\right)^{1 / 2}} \frac{\partial \chi}{\partial s}+\frac{\phi_{u}}{\sin \omega\left(\Delta_{1} \phi\right)^{1 / 2}} \frac{\partial \chi}{\partial s^{\prime}}, \\
& \chi_{v}=-\frac{\psi_{v}}{\sin \omega\left(\Delta_{1} \psi\right)^{1 / 2}} \frac{\partial \chi}{\partial s}+\frac{\phi_{v}}{\sin \omega\left(\Delta_{1} \phi\right)^{1 / 2}} \frac{\partial \chi}{\partial s^{\prime}} .
\end{aligned}
$$

Forming $d \chi$ from these relations and introducing $d s$ and $d s^{\prime}$ from (10), we find

$$
d \chi=\frac{\partial \chi}{\partial s} d s+\frac{\partial \chi}{\partial s^{\prime}} d s^{\prime}
$$

On the other hand, if we differentiate the first of the equations (12) with respect to $v$ and the second with respect to $u$ and equate the resulting expressions, the equation obtained reduces to

$$
\frac{\partial}{\partial s^{\prime}} \frac{\partial \chi}{\partial s}-\frac{\partial}{\partial s} \frac{\partial \chi}{\partial s^{\prime}}=\frac{1}{\sigma} \frac{\partial \chi}{\partial s}+\frac{1}{\sigma^{\prime}} \frac{\partial \chi}{\partial s^{\prime}},
$$

where

(15) $\frac{1}{\sigma}=\frac{\partial}{\partial s^{\prime}} \log \left(\sin \omega\left(\Delta_{1} \psi\right)^{1 / 2}\right), \frac{1}{\sigma^{\prime}}=-\frac{\partial}{\partial s} \log \left(\sin \omega\left(\Delta_{1} \phi\right)^{1 / 2}\right)$.

A necessary and sufficient condition that two functions of $u$ and $v$, which we denote by $\partial \chi / \partial s$ and $\partial \chi / \partial s^{\prime}$, be the directional derivatives 
in the directions of the curves $C$ and $C^{\prime}$ of a function $\chi(u, v)$ is that

$$
\frac{\partial}{\partial s^{\prime}} \frac{\partial \chi}{\partial s}-\frac{\partial}{\partial s} \frac{\partial \chi}{\partial s^{\prime}}=\frac{1}{\sigma} \frac{\partial \chi}{\partial s}+\frac{1}{\sigma^{\prime}} \frac{\partial \chi}{\partial s^{\prime}} .
$$

When the condition is satisfied,

$$
\chi=\int \frac{\partial \chi}{\partial s} d s+\frac{\partial \chi}{\partial s^{\prime}} d s^{\prime} .
$$

6. The Invariants $1 / \sigma, 1 / \sigma^{\prime}$. In seeking the geometric interpretations of the invariants $1 / \sigma$ and $1 / \sigma^{\prime}$ given by (15), we consider first the significance of their vanishing.*

The curves $C^{\prime}$ are equidistant curves with respect to the curves $C$, that is, every two curves $C^{\prime}$ cut segments of equal length from the curves $C$, if and only if the invariant $1 / \sigma$ is zero.

For, if $1 / \sigma=0$, the function $\sin \omega\left(\Delta_{1} \psi\right)^{1 / 2}$ is constant along each curve $C^{\prime}$ and is, therefore, a function of $\psi$. Then, $d s$, as given by $(10)$, is actually the exact differential of a function $s(\psi)$, which is the common arc of the curves $C$ measured from a fixed curve $C^{\prime}$ to an arbitrary curve $C^{\prime}$. The argument may be reversed.

The geometric interpretation of

$$
\frac{1}{\sigma}=\frac{\partial}{\partial s^{\prime}} \log \left(\sin \omega\left(\Delta_{1} \psi\right)^{1 / 2}\right)
$$

is now clear. It is a measure of the deviation from one another of the curves $C^{\prime}$ with respect to the curves $C$. When we think of $d \psi$ in (10) as having been given a fixed value, it is precisely, except for sign, the logarithmic rate of change, in the direction of the general curve $C^{\prime}$, of the element of arc of the general curve $C$. We shall call it the relative variation of the element of arc of the curve $C$ with respect to the curve $C^{\prime} . \dagger$

The relative variation, $1 / \sigma$, of the element of arc of $C$ with respect to $C^{\prime}$ is taken in the positive direction of $C^{\prime}$, that is, in the direction on $C^{\prime}$ to the left of $C$ in the sense described in $\$ 5$.

* The significance of the vanishing of $1 / \sigma$ and $1 / \sigma^{\prime}$ is well known; see Lilienthal, Vorlesungen, vol. 2, p. 228.

$\dagger$ It is to be noted that $1 / \sigma$ is unchanged when $\psi$ is replaced by $F(\psi)$. This is not true, for example, of $\left(\partial / \partial s^{\prime}\right)\left(\sin \omega\left(\Delta_{1} \psi\right)^{1 / 2}\right)$. 
Accordingly, we should take the relative variation, $1 / \sigma^{\prime}$, of the element of arc of $C^{\prime}$ with respect to $C$ in the direction on $C$ to the left of $C^{\prime}$. This is the negative direction on $C$. Hence, we should have, and, by (15), actually do have

$$
\frac{1}{\sigma^{\prime}}=-\frac{\partial}{\partial s} \log \left(\sin \omega\left(\Delta_{1} \phi\right)^{1 / 2}\right) \text {. }
$$

From the law of change, with the direction, of the directional derivative, it is evident that

$$
\sin \omega\left(\Delta_{1} \psi\right)^{1 / 2}=-\frac{\partial \psi}{\partial s}, \quad \sin \omega\left(\Delta_{1} \phi\right)^{1 / 2}=\frac{\partial \phi}{\partial s^{\prime}} .
$$

Hence, we get the formulas

$$
\frac{1}{\sigma}=\frac{\partial}{\partial s^{\prime}} \log \left(-\frac{\partial \psi}{\partial s}\right), \frac{1}{\sigma^{\prime}}=-\frac{\partial}{\partial s} \log \left(\frac{\partial \psi}{\partial s^{\prime}}\right),
$$

which give new interpretations of $1 / \sigma$ and $1 / \sigma^{\prime}$.

We suppose now that the curves $C$ and $C^{\prime}$ form an orthogonal system: $\omega=\pi / 2$, and compute their geodesic curvatures, $1 / \rho$ and $1 / \rho^{\prime}$. Substituting for $x_{u}$ and $x_{v}$ in (7a) their values in terms of $\xi$ and $\xi^{\prime}$, and setting, for $\partial x / \partial s, \xi$ and $\xi^{\prime}$ in turn, we find

$$
\begin{aligned}
& \frac{1}{\rho}=\frac{1}{D}\left[\frac{\partial}{\partial u}\left(-\frac{\psi_{v}}{\left(\Delta_{1} \psi\right)^{1 / 2}}\right)-\frac{\partial}{\partial v}\left(-\frac{\psi_{u}}{\left(\Delta_{1} \psi\right)^{1 / 2}}\right)\right], \\
& \frac{1}{\rho^{\prime}}=\frac{1}{D}\left[\frac{\partial}{\partial u}\left(\frac{\phi_{v}}{\left(\Delta_{1} \phi\right)^{1 / 2}}\right)-\frac{\partial}{\partial v}\left(\frac{\phi_{u}}{\left(\Delta_{1} \phi\right)^{1 / 2}}\right)\right] .
\end{aligned}
$$

Hence, we have

(16a) $\frac{1}{\rho}=\frac{\partial}{\partial s^{\prime}} \log \left(\Delta_{1} \psi\right)^{1 / 2}=\frac{1}{\sigma}, \frac{1}{\rho^{\prime}}=-\frac{\partial}{\partial s} \log \left(\Delta_{1} \phi\right)^{1 / 2}=\frac{1}{\sigma^{\prime}}$.

If the curves $C$ and $C^{\prime}$ form an orthogonal system, the relative variations of their elements of arc, $1 / \sigma$ and $1 / \sigma^{\prime}$, are equal respectively to their geodesic curvatures, $1 / \rho$ and $1 / \rho^{\prime}$.

We thus have new interpretations of the geodesic curvatures of the curves of an orthogonal system.

7. The Modified Directional Derivatives. The condition of integrability (14) tells us that the order of the operations repre- 
sented by the symbols $\partial / \partial s$ and $\partial / \partial s^{\prime}$ cannot be inverted. We propose to introduce modifications of the directional derivatives $\partial / \partial s, \partial / \partial s^{\prime}$, which, when used in conjunction with these derivatives, will not only remedy this defect, but also render our methods more powerful and elegant.

The modifications of $\partial / \partial s$ and $\partial / \partial s^{\prime}$, which we shall den ote by $\nabla / \nabla s$ and $\nabla / \nabla s^{\prime}$, are defined as follows:*

$$
\frac{\nabla \chi}{\nabla s}=\frac{\partial \chi}{\partial s}+\frac{1}{\sigma^{\prime}} \chi, \frac{\nabla \chi}{\nabla s^{\prime}}=\frac{\partial \chi}{\partial s^{\prime}}-\frac{1}{\sigma} \chi .
$$

According to the definition,

$$
\frac{\nabla}{\nabla s^{\prime}} \frac{\partial \chi}{\partial s}=\frac{\partial}{\partial s^{\prime}} \frac{\partial \chi}{\partial s}-\frac{1}{\sigma} \frac{\partial \chi}{\partial s}, \quad \frac{\nabla}{\nabla s} \frac{\partial \chi}{\partial s^{\prime}}=\frac{\partial}{\partial s} \frac{\partial \chi}{\partial s^{\prime}}+\frac{1}{\sigma^{\prime}} \frac{\partial \chi}{\partial s^{\prime}} .
$$

Hence, the condition of integrability (14) becomes

$$
\frac{\nabla}{\nabla s^{\prime}} \frac{\partial \chi}{\partial s}=\frac{\nabla}{\nabla s} \frac{\partial \chi}{\partial s^{\prime}}
$$

$A$ necessary and sufficient condition that $Q(u, v) d s+Q^{\prime}(u, v) d s^{\prime}$ be an exact differential is that

$$
\frac{\nabla Q}{\nabla s^{\prime}}=\frac{\nabla Q^{\prime}}{\nabla s}
$$

The modified derivative of a sum is the sum of the modified derivatives. But the standard law for differentiating a product does not hold for the modified differentiation. We have, instead,

$$
\frac{\nabla\left(\chi_{1} \chi_{2}\right)}{\nabla s}=\chi_{1} \frac{\nabla \chi_{2}}{\nabla s}+\chi_{2} \frac{\partial \chi_{1}}{\partial s}=\chi_{1} \frac{\partial \chi_{2}}{\partial s}+\chi_{2} \frac{\nabla \chi_{1}}{\nabla s} .
$$

The fact that one of the factors is subjected to ordinary differentiation, and the other to the modified differentiation, works out advantageously in practice, as will be seen later.

When the given system of curves is an orthogonal system, $1 / \sigma=1 / \rho$ and $1 / \sigma^{\prime}=1 / \rho^{\prime}$. Consequently, in this case, both

* Though the author came upon it otherwise, the reader will find the germ of the idea of the modified directional derivative in the abbreviations $\left(\partial / \partial s+1 / \rho^{\prime}\right) \chi$ and $\left(\partial / \partial s^{\prime}-1 / \rho\right) \chi$ employed by Cesàro, loc. cit., in the discussion of the case in which the curves $C$ and $C^{\prime}$ form an orthogonal system. 
modified directional derivatives are identical with the corresponding ordinary derivatives if and only if $S$ is a plane and toe system of curves consists of two orthogonal pencils of straight lines.

On the other hand, in the general case, both modified derivatives coincide with the corresponding ordinary derivatives for every system of curves which clothes $S$ in the sense of Tchebycheff. For $1 / \sigma$ and $1 / \sigma^{\prime}$ both vanish if and only if the curves of each family of the system are equidistant with respect to the curves of the other family, and this is a characteristic property of a system of curves which clothes the surface.

C. The Canonical Differential Equations of the Families of Curves of a System

8. The Canonical Differential Equations, Derived from Finite Equations. In connection with the system of curves consisting of the family of curves $C: \phi(u, v)=$ const. and the family of cvrves $C^{\prime}: \psi(u, v)=$ const., we recall the expressions

$$
d s=-\frac{d \psi}{\sin \omega\left(\Delta_{1} \psi\right)^{1 / 2}}, \quad d s^{\prime}=\frac{d \phi}{\sin \omega\left(\Delta_{1} \phi\right)^{1 / 2}}
$$

for the differentials of arc of the curves $C$ and $C^{\prime}$.

When we set

$$
\begin{array}{ll}
A^{\prime}=-\frac{\psi_{u}}{\sin \omega\left(\Delta_{1} \psi\right)^{1 / 2}}, \quad B^{\prime}=-\frac{\psi_{v}}{\sin \omega\left(\Delta_{1} \psi\right)^{1 / 2}}, \\
A=\frac{\phi_{u}}{\sin \omega\left(\Delta_{1} \phi\right)^{1 / 2}}, \quad B=\frac{\phi_{v}}{\sin \omega\left(\Delta_{1} \phi\right)^{1 / 2}},
\end{array}
$$

these expressions become

$$
d s=A^{\prime} d u+B^{\prime} d v, \quad d s^{\prime}=A d u+B d v .
$$

Since $\phi, \psi, \Delta_{1} \phi$, and $\Delta_{1} \psi$ are absolute invariants with respect to a change of parameters, and $\sin \omega$ is an absolute invariant except perhaps for a change of signs, the linear differential forms $A d u+B d v$ and $A^{\prime} d u+B^{\prime} d v$ are absolute invariants except perhaps for a change of signs.

It is evident that

$$
A d u+B d v=0, \quad A^{\prime} d u+B^{\prime} d v=0
$$


are differential equations, respectively, of the families of curves $C$ and $C^{\prime}$. We shall call them the canonical differential equations of these families and employ them exclusively, from now on, to represent the families.

The presence of the factor $\sin \omega$ in formulas (10) shows that a canonical differential equation of a family of curves $C$ is determined only when there is associated with the family $C$ a second family $C^{\prime}$. In other words, the concept of canonical differential equations applies only to two families of curves forming a system, and not to individual families of curves.

We proceed now to express the theory thus far developed in terms of canonical differential equations and the coefficients $A, B, A^{\prime}, B^{\prime}$ contained in them.

From (9) we have, as the new form of the linear element,

$$
(d x \mid d x)=d s^{2}+2 \cos \omega d s d s^{\prime}+d s^{\prime 2} .
$$

According to (8),

$$
\xi=\frac{\sin \omega}{D}\left(B x_{u}-A x_{v}\right), \quad \xi^{\prime}=-\frac{\sin \omega}{D}\left(B^{\prime} x_{u}-A^{\prime} x_{v}\right) .
$$

Substituting these expressions in to

we find

$$
\tilde{\xi \xi^{\prime}}=\zeta \sin \omega
$$

$$
D=\sin \omega\left(A^{\prime} B-A B^{\prime}\right) \text {. }
$$

Hence, when we set

$$
\bar{D}=A^{\prime} B-A B^{\prime}
$$

we have

$$
D=\bar{D} \sin \omega .
$$

The expressions for $\xi$ and $\xi^{\prime}$ now become

$$
\xi=\frac{1}{\bar{D}}\left(B x_{u}-A x_{v}\right), \quad \xi^{\prime}=-\frac{1}{\bar{D}}\left(B^{\prime} x_{u}-A^{\prime} x_{v}\right) .
$$

Similarly, we have

$$
\frac{\partial \chi}{\partial s}=\frac{1}{\bar{D}}\left(B \chi_{u}-A \chi_{v}\right), \quad \frac{\partial \chi}{\partial s^{\prime}}=-\frac{1}{\bar{D}}\left(B^{\prime} \chi_{u}-A^{\prime} \chi_{v}\right) .
$$

The condition of integrability is of the same form as before, 
where now the values of $1 / \sigma$ and $1 / \sigma^{\prime}$ are given by the much simpler expressions

$$
\frac{1}{\sigma}=\frac{1}{\bar{D}}\left(B_{u}^{\prime}-A_{v}^{\prime}\right), \quad \frac{1}{\sigma^{\prime}}=\frac{1}{\bar{D}}\left(B_{u}-A_{v}\right) .
$$

From (24) and (25) it is readily seen that the modified direction'al derivatives have the values

$$
\begin{aligned}
& \frac{\nabla \chi}{\nabla s}=\frac{\partial \chi}{\partial s}+\frac{1}{\sigma^{\prime}} \chi=\frac{1}{\bar{D}}\left[(B \chi)_{u}-(A \chi)_{v}\right], \\
& \frac{\nabla \chi}{\nabla s^{\prime}}=\frac{\partial \chi}{\partial s^{\prime}}-\frac{1}{\sigma} \chi=-\frac{1}{\bar{D}}\left[\left(B^{\prime} \chi\right)_{u}-\left(A^{\prime} \chi\right)_{v}\right] .
\end{aligned}
$$

Thus, the vanishing of $\nabla \chi / \nabla s$ is a condition necessary and sufficient that $\chi$ be an integrating factor of $A d u+B d v$, and the vanishing of $\nabla \chi / \nabla s^{\prime}$ is a necessary and sufficient condition that $\chi$ be an integrating factor of $A^{\prime} d u+B^{\prime} d v$.* $^{*}$

9. Derivation of Canonical Differential Equations from Arbitrary Differential Equations. The canonical differential equations of the families of curves $C$ and $C^{\prime}$ are readily found, as we have seen, when the families of curves are defined by finite equations. They may be found with equal ease, without quadratures, when the families of curves are defined by differential equations, chosen at random.

Let differential equations defining the curves $C$ and $C^{\prime}$ be

$$
C: M d u+N d v=0, \quad C^{\prime}: \quad M^{\prime} d u+N^{\prime} d v=0 .
$$

We may assume that $M N^{\prime}-N M^{\prime}>0$, since otherwise we could change the signs of, say, $M^{\prime}$ and $N^{\prime}$. Let, then, $I(u, v)>0$ and $I^{\prime}(u, v)>0$ denote positive integrating factors of $M d u+N d v$ and $M^{\prime} d u+N^{\prime} d v$, respectively, and form

* The corresponding conditions in terms of the ordinary derivatives are, respectively,

$$
\frac{\partial \log \chi}{\partial s}=-\frac{1}{\sigma^{\prime}}, \frac{\partial \log \chi}{\partial s^{\prime}}=\frac{1}{\sigma},
$$

and these conditions are in keeping with the geometric interpretations of $1 / \sigma$ and $1 / \sigma^{\prime}$. In particular, when $1 / \sigma$ and $1 / \sigma^{\prime}$ are both zero, the linear forms $A^{\prime} d u+B^{\prime} d v$ and $A d u+B d v$ are exact differentials, $s$ and $s^{\prime}$ are parameters for the surface, and the linear element (21) becomes characteristic for a system of curves which clothes the surface. 


$$
d \phi=I M d u+I N d v, \quad d \psi=I^{\prime} M^{\prime} d u+I^{\prime} N^{\prime} d v .
$$

Then $[\phi, \psi]>0$ and $\sin \omega>0$.

The quantities $A, B, A^{\prime}, B^{\prime}$ may now be computed from (27), by means of (19), and, what is more important, the values obtained for them are independent of $I$ and $I^{\prime}$.

\section{The Surface Referred to an Orthogonal System of Curves}

10. The Differential Equations of the Vectors of the Trihedral $\xi, \xi^{\prime}, \zeta$. Let the canonical differential equations of the families of curves $C$ and $C^{\prime}$ of an orthogonal system* on $S$ be, respectively,

$$
A d u+B d v=0, \quad A^{\prime} d u+B^{\prime} d v=0 .
$$

The important formulas for this case may be obtained from those of $\$ 8$ by setting $\sin \omega=1, \bar{D}=D$, and replacing $1 / \sigma$ and $1 / \sigma^{\prime}$ by $1 / \rho$ and $1 / \rho^{\prime}$.

Thus, the unit vectors $\xi$ and $\xi^{\prime}$ in the positive directions of the curves $C$ and $C^{\prime}$ are

$$
\xi=\frac{1}{D}\left(B x_{u}-A x_{v}\right), \quad \xi^{\prime}=-\frac{1}{D}\left(B^{\prime} x_{u}-A^{\prime} x_{v}\right),
$$

where

Hence

$$
D=A^{\prime} B-A B^{\prime}
$$

$$
x_{u}=A^{\prime} \xi+A \xi^{\prime}, \quad x_{v}=B^{\prime} \xi+B \xi^{\prime} .
$$

For the geodesic curvatures of $C$ and $C^{\prime}$ and the directional derivatives in their positive directions, we have

$$
\begin{aligned}
& \frac{1}{\rho}=\frac{1}{D}\left(B_{u}^{\prime}-A_{v}^{\prime}\right), \quad \frac{1}{\rho^{\prime}}=\frac{1}{D}\left(B_{u}-A_{v}\right), \\
& \frac{\partial \chi}{\partial s}=\frac{1}{D}\left(B \chi_{u}-A \chi_{v}\right), \quad \frac{\partial \chi}{\partial s^{\prime}}=-\frac{1}{D}\left(B^{\prime} \chi_{u}-A^{\prime} \chi_{v}\right), \\
& \frac{\nabla \chi}{\nabla s}=\frac{1}{D}\left[(B \chi)_{u}-(A \chi)_{v}\right]=\frac{\partial \chi}{\partial s}+\frac{1}{\rho^{\prime}} \chi \\
& \frac{\nabla \chi}{\nabla s^{\prime}}=-\frac{1}{D^{\prime}}\left[\left(B^{\prime} \chi\right)_{u}-\left(A^{\prime} \chi\right)_{v}\right]=\frac{\partial \chi}{\partial s^{\prime}}-\frac{1}{\rho} \chi,
\end{aligned}
$$

* For the further development of the treatment of the surface referred to an arbitrary system of curves, see author, loc. cit. 
where

$$
D=A^{\prime} B-A B^{\prime} .
$$

We also have

where

$$
d \chi=\frac{\partial \chi}{\partial s} d s+\frac{\partial \chi}{\partial s^{\prime}} d s^{\prime}
$$

$$
d s=A^{\prime} d u+B^{\prime} d v, \quad d s^{\prime}=A d u+B d v .
$$

The unit vectors $\xi, \xi^{\prime}$, in the positive directions of curves $C, C^{\prime}$, and the unit vector $\zeta$ normal to the surface form a rectangular trihedral which has the same disposition as the axes: $\left(\xi \xi^{\prime} \zeta\right)=1$. We propose to find expressions for the directional derivatives, in the directions of the curves $C$ and $C^{\prime}$, of the vectors of this trihedral, and to write them as linear combinations of these vectors. Since $\xi, \xi^{\prime}, \zeta$ are mutually orthogonal unit vectors, the determinant of the coefficients in the linear combinations which are respectively the values of the directional derivatives of $\xi, \xi^{\prime}, \zeta$ in a specific direction is skewsymmetric.

We obtain the values of $\partial \xi / \partial s, \partial \xi^{\prime} / \partial s, \partial \zeta / \partial s$ by considering an arbitrary curve $C$. The geodesic curvature of $C$ is

$$
\frac{1}{\rho}=\frac{\left(\eta \mid \xi^{\prime}\right)}{R}
$$

where $\eta$ is the unit vector in the positive direction of the principal normal to $C$, and $1 / R$ is the curvature of $C$. But $\eta / R=\partial \xi / \partial s$, and hence

$$
\frac{1}{\rho}=\left(\frac{\partial \xi}{\partial s} \mid \xi^{\prime}\right)=-\left(\frac{\partial \xi^{\prime}}{\partial s} \mid \xi\right)
$$

where the second of the two values results from the fact that $\left(\xi \mid \xi^{\prime}\right)=0$.

The normal curvature of $C$ is given by the formula

$$
\frac{1}{r}=-\frac{(d x \mid d \zeta)}{(d x \mid d x)}=\frac{e d u^{2}+2 f d u d v+g d v^{2}}{E d u^{2}+2 F d u d v+G d v^{2}} .
$$

Since, along $C,(d x \mid d x)=d s^{2}$ and $\partial x / \partial s=\xi$,

$$
\frac{1}{r}=-\left(\frac{\partial \zeta}{\partial s} \mid \xi\right)=\left(\frac{\partial \xi}{\partial s} \mid \zeta\right)
$$


The familiar formula,

$$
\frac{1}{\tau}=-\frac{(E f-F e) d u^{2}+(E g-G e) d u d v+(F g-G f) d v^{2}}{D\left(E d u^{2}+2 F d u d v+G d v^{2}\right)}
$$

for the geodesic curvature of $C$ amounts to nothing more than

$$
\frac{1}{\tau}=-\left(\frac{\partial \zeta}{\partial s} \frac{\partial x}{\partial s} \zeta\right)
$$

But $\partial x / \partial s=\xi$ and $\widetilde{\xi \zeta}=-\xi^{\prime}$. Hence

$$
\frac{1}{\tau}=\left(\frac{\partial \zeta}{\partial s} \mid \xi^{\prime}\right)=-\left(\frac{\partial \xi^{\prime}}{\partial s} \mid \zeta\right)
$$

Formulas (31), (32), (33) are equivalent to

$$
\begin{aligned}
& \frac{\partial \xi}{\partial s}= \\
& \frac{\partial \xi^{\prime}}{\partial s}=-\frac{1}{\rho} \xi^{\prime}+\frac{1}{r} \zeta, \\
& \frac{\partial \zeta}{\partial s}=-\frac{1}{r} \xi+\frac{1}{\tau} \xi^{\prime} .
\end{aligned}
$$

The trihedral for the general curve $C^{\prime}$ which corresponds to the trihedral $\xi, \xi^{\prime}, \zeta$ for the curve $C$ is $\xi^{\prime}, \xi^{\prime \prime}=-\xi, \zeta$. Hence,

$$
\begin{array}{ll}
\frac{\partial \xi^{\prime}}{\partial s^{\prime}}= & -\frac{1}{\rho^{\prime}} \xi+\frac{1}{r^{\prime}} \zeta, \\
\frac{\partial \xi}{\partial s^{\prime}}=\frac{1}{\rho^{\prime}} \xi^{\prime} \quad+\frac{1}{\tau^{\prime}} \zeta, \\
\frac{\partial \zeta}{\partial s}=\frac{1}{r^{\prime}} \xi^{\prime}-\frac{1}{\tau^{\prime}} \xi
\end{array}
$$

where $1 / \rho^{\prime}, 1 / r^{\prime}, 1 / \tau^{\prime}$ are respectively the geodesic curvature, the normal curvature, and the geodesic torsion of the curve $C^{\prime}$.

Consider, now, the condition of integrability (14) of the equations

$$
\frac{\partial x}{\partial s}=\xi, \quad \frac{\partial x}{\partial s^{\prime}}=\xi^{\prime}
$$


namely,

$$
\frac{\partial \xi}{\partial s^{\prime}}-\frac{\partial \xi^{\prime}}{\partial s}=\frac{1}{\rho} \xi+\frac{1}{\rho^{\prime}} \xi^{\prime} .
$$

When for $\partial \xi / \partial s^{\prime}$ and $\partial \xi^{\prime} / \partial s$ are substituted their values from (34) and (35), this condition reduces to

$$
\frac{1}{\tau^{\prime}}=-\frac{1}{\tau}
$$

Thus, the required system of differential equations for the vectors $\xi, \xi^{\prime}, \zeta$ is

$$
\begin{aligned}
\frac{\partial \xi}{\partial s} & = & \frac{1}{\rho} \xi^{\prime}+\frac{1}{r} \zeta, \frac{\partial \xi}{\partial s^{\prime}} &
\end{aligned}
$$

We know that

$$
d x=\xi d s+\xi^{\prime} d s^{\prime} .
$$

By the last pair of equations in (38),

$$
-d \zeta=\left(\frac{1}{r} \xi-\frac{1}{\tau} \xi^{\prime}\right) d s+\left(-\frac{1}{\tau} \xi+\frac{1}{r^{\prime}} \xi^{\prime}\right) d s^{\prime} .
$$

Hence

$$
\begin{aligned}
(d x \mid d x) & =d s^{2}+d s^{\prime 2} \\
(39)-(d x \mid d \zeta) & =\frac{1}{r} d s^{2}-\frac{2}{\tau} d s d s^{\prime}+\frac{1}{r^{\prime}} d s^{\prime 2}, \\
(d \zeta \mid d \zeta) & =\left(\frac{1}{r^{2}}+\frac{1}{\tau^{2}}\right) d s^{2}-\frac{2}{\tau} K^{\prime} d s d s^{\prime}+\left(\frac{1}{r^{\prime 2}}+\frac{1}{\tau^{\prime 2}}\right) d s^{\prime 2} .
\end{aligned}
$$

The three fundamental quadratic forms of the surface are expressible in terms of (a) the canonical linear forms, $d s^{\prime}=A d u+B d v$ and $d s=A^{\prime} d u+B^{\prime} d v$, and (b) the normal curvatures, $1 / r, 1 / r^{\prime}$, and geodesic torsion, $1 / \tau$, associated with the curves $C$ and $C^{\prime}$. 
It is evident that we have now all the advantages of having the surface referred to the given orthogonal system of curves, while the parametric curves themselves remain arbitrary.

11. The Fundamental Theorem. The relation (37), which expresses the fact that equations (36) are compatible, has already been taken account of in equations (38).

If we apply the condition of integrability, in the form

$$
\frac{\nabla}{\nabla s^{\prime}} \frac{\partial \chi}{\partial s}=\frac{\nabla}{\nabla s} \frac{\partial \chi}{\partial s^{\prime}}
$$

to the directional derivatives of $\xi, \xi^{\prime}, \zeta$ in (38), we obtain the following relations :*

$$
\begin{gathered}
\frac{\nabla}{\nabla s^{\prime}}\left(\frac{1}{\rho}\right)-\frac{\nabla}{\nabla s}\left(\frac{1}{\rho^{\prime}}\right)=K, \text { where } K=\frac{1}{r r^{\prime}}-\frac{1}{\tau^{2}}, \\
\frac{\nabla}{\nabla s^{\prime}}\left(\frac{1}{r}\right)+\frac{\nabla}{\nabla s}\left(\frac{1}{\tau}\right)+\frac{1}{\rho} \frac{1}{r^{\prime}}+\frac{1}{\rho^{\prime}} \frac{1}{\tau}=0, \\
\frac{\nabla}{\nabla s}\left(\frac{1}{r^{\prime}}\right)+\frac{\nabla}{\nabla s^{\prime}}\left(\frac{1}{\tau}\right)-\frac{1}{\rho^{\prime}} \frac{1}{r}-\frac{1}{\rho} \frac{1}{\tau}=0 .
\end{gathered}
$$

Equation (40) is the Gauss equation, expressed invariantly with reference to the given orthogonal system, and equations (41) are the equations of Codazzi, in similar form.

It is clear from (30) that equations (40) and (41) are equations which connect the seven fundamental quantities, $A, B$, $A^{\prime}, B^{\prime}, 1 / r, 1 / r^{\prime}, 1 / \tau$, and their partial derivatives with respect to $u$ and $v$. They constitute necessary conditions for the existence of a surface $x=x(u, v)$ for which the equations $A d u+B d v=0$ and $A^{\prime} d u+B^{\prime} d v=0$ define an orthogonal system of curves $C$ and $C^{\prime}$ whose normal curvatures and geodesic torsions are $1 / r, 1 / r^{\prime}$ and $1 / \tau,-1 / \tau$.

* In applying the condition, we have to find the modified derivatives of products of the form $k \delta$, where $k$ is a function and $\delta$ is one of the vectors $\xi, \xi^{\prime}, \zeta$. To one factor of the product we must apply the ordinary differentiation, and to the other, the modified differentiation. The advantage of this is evident. For, we may take $\delta$ as the factor to which to apply the ordinary differentiation, and the ordinary derivatives of $\xi, \xi^{\prime}, \zeta$ are just what are given us in (38). 
These conditions are also sufficient. For, if the seven quantities in question satisfy equations (40) and (41), the system of differential equations (38), subject to the conditions

$(\xi \mid \xi)=\left(\xi^{\prime} \mid \xi^{\prime}\right)=(\zeta \mid \zeta)=1,\left(\xi \mid \xi^{\prime}\right)=(\xi \mid \zeta)=\left(\xi^{\prime} \mid \zeta\right)=0,\left(\xi \xi^{\prime} \zeta\right)=1$,

is integrable. The resulting rectangular trihedral of unit vectors, $\xi, \xi^{\prime}, \zeta$, is determined to within a rigid motion.

When the expressions found for $\xi$ and $\xi^{\prime}$ are substituted.in equations (36), these equations are integrable. A surface,

$$
x=\int \xi d s+\xi^{\prime} d s^{\prime},
$$

is thus determined to within its position in space. Since the three fundamental forms of this surface are as given in (39), it is readily shown to have the desired properties.

If two linear differential forms, $A d u+B d v$ and $A^{\prime} d u+B^{\prime} d v$, and three functions, $1 / r, 1 / r^{\prime}$, and $1 / \tau$, are given, such that equations (40) and (41), where

$$
\begin{array}{ll}
\frac{1}{\rho}=\frac{1}{D}\left(B_{u}^{\prime}-A_{v}^{\prime}\right), & \frac{1}{\rho^{\prime}}=\frac{1}{D}\left(B_{u}-A_{v}\right), \\
\frac{\nabla \chi}{\nabla s}=\frac{1}{D}\left[(B \chi)_{u}-(A \chi)_{v}\right], & \frac{\nabla \chi}{\nabla s^{\prime}}=-\frac{1}{D}\left[\left(B^{\prime} \chi\right)_{u}-\left(A^{\prime} \chi\right)_{v}\right],
\end{array}
$$

and

$$
D=A^{\prime} B-A B^{\prime} \neq 0,
$$

are satisfied, there exists a surface, unique to within its fosition in space, on which the equations $A d u+B d v=0, A^{\prime} d u+B^{\prime} d v=0$ define two orthogonal families of curves which have $1 / r, 1 / r^{\prime}$ as their normal curvatures, and $1 / \tau,-1 / \tau$ as their geodesic torsions.

We permit ourselves, at this point, one application. The equation $K=0$, that is,

$$
\frac{\nabla}{\nabla s^{\prime}}\left(\frac{1}{\rho}\right)=\frac{\nabla}{\nabla s}\left(\frac{1}{\rho^{\prime}}\right),
$$

says that the expression

$$
\frac{d s}{\rho}+\frac{d s^{\prime}}{\rho^{\prime}}
$$


is an exact differential. Thus, there exists a function, $\theta(u, v)$, for which

$$
\frac{\partial \theta}{\partial s}=\frac{1}{\rho}, \quad \frac{\partial \theta}{\partial s^{\prime}}=\frac{1}{\rho^{\prime}} .
$$

But these are precisely the conditions that the expressions

$$
\begin{array}{r}
e^{\theta i}\left[\left(A-i A^{\prime}\right) d u+\left(B-i B^{\prime}\right) d v\right], \\
e^{-\theta i}\left[\left(A+i A^{\prime}\right) d u+\left(B+i B^{\prime}\right) d v\right],
\end{array}
$$

be exact differentials, as can be immediately verified. The product of the two expressions can, therefore, be written in the form $d u_{1}^{2}+d v_{1}^{2}$. But this product is the linear element of the surface.

Thus, we have established, in a simple and elegant manner, a theorem whose proof by the usual methods is decidedly laborious, namely, the theorem to the effect that the linear element of a developable surface can be reduced by quadratures to the form $d u^{2}+d v^{2}$.

12. The Surface Referred to the Lines of Curvature. We shall denote the curves of the two families of lines of curvature by $C_{1}$ and $C_{2}$, the canonical differential equations which represent them by

$$
A_{1} d u+B_{1} d v=0, \quad A_{2} d u+B_{2} d v=0,
$$

and the unit vectors in their positive directions, by $\xi^{(1)}, \xi^{(2)}$.

The following formulas are, then, self-explanatory:

$$
D=A_{2} B_{1}-A_{1} B_{2},
$$

$$
\frac{1}{\rho_{1}}=\frac{1}{D}\left(\frac{\partial B_{2}}{\partial u}-\frac{\partial A_{2}}{\partial v}\right), \quad \frac{1}{\rho_{2}}=\frac{1}{D}\left(\frac{\partial B_{1}}{\partial u}-\frac{\partial A_{1}}{\partial v}\right),
$$

(42) $\frac{\partial \chi}{\partial s_{1}}=\frac{1}{D}\left(B_{1} \chi_{u}-A_{1} \chi_{v}\right), \quad \frac{\partial \chi}{\partial s_{2}}=-\frac{1}{D}\left(B_{2} \chi_{u}-A_{2} \chi_{v}\right)$,

$$
\frac{\nabla \chi}{\nabla s_{1}}=\frac{1}{D}\left[\left(B_{1} \chi\right)_{u}-\left(A_{1} \chi\right)_{v}\right], \frac{\nabla \chi}{\nabla s_{2}}=-\frac{1}{D}\left[\left(B_{2} \chi\right)_{u}-\left(A_{2} \chi\right)_{v}\right] .
$$

Since the lines of curvature are characterized by the vanishing 
of the geodesic torsion, the fundamental differential forms of $S$ are, in this case,

$$
d s_{1}^{2}+d s_{2}^{2}, \quad \frac{1}{r_{1}} d s_{1}^{2}+\frac{1}{r_{2}} d s_{2}^{2}, \quad \frac{1}{r_{1}^{2}} d s_{1}^{2}+\frac{1}{r_{2}^{2}} d s_{2}{ }^{2},
$$

and the fundamental differential equations,

$$
\begin{aligned}
& \frac{\partial x}{\partial s_{1}}=\xi^{(1)}, \quad \frac{\partial x}{\partial s_{2}}=\xi^{(2)}, \\
& \frac{\partial \xi^{(1)}}{\partial s_{1}}=\frac{1}{\rho_{1}} \xi^{(2)}+\frac{1}{r_{1}} \zeta, \quad \frac{\partial \xi^{(1)}}{\partial s_{2}}=\frac{1}{\rho_{2}} \xi^{(2)}, \\
& \frac{\partial \xi^{(2)}}{\partial s_{1}}=-\frac{1}{\rho_{1}} \xi^{(1)}, \quad \frac{\partial \xi^{(2)}}{\partial s_{2}}=-\frac{1}{\rho_{2}} \xi^{(1)}+\frac{1}{r_{2}} \zeta, \\
& \frac{\partial \zeta}{\partial s_{1}}=-\frac{1}{r_{1}} \xi^{(1)}, \quad \frac{\partial \zeta}{\partial s_{2}}=-\frac{1}{r_{2}} \xi^{(2)} .
\end{aligned}
$$

The Gauss-Codazzi equations take the simple forms

$$
\begin{array}{ll}
\frac{\nabla}{\nabla s_{2}}\left(\frac{1}{\rho_{1}}\right)-\frac{\nabla}{\nabla s_{1}}\left(\frac{1}{\rho_{2}}\right)=K, & K=\frac{1}{r_{1} r_{2}}, \\
\frac{\nabla}{\nabla s_{2}}\left(\frac{1}{r_{1}}\right)+\frac{1}{\rho_{1}} \frac{1}{r_{2}}=0, & \frac{\nabla}{\nabla s_{1}}\left(\frac{1}{r_{2}}\right)-\frac{1}{\rho_{2}} \frac{1}{r_{1}}=0 .
\end{array}
$$

The fundamental theorem is also simpler, since it bears merely on the six functions $A_{1}, B_{1}, A_{2}, B_{2}, 1 / r_{1}, 1 / r_{2}$.

13. A Complete System of Invariant Vectors. We proceed to establish the following theorem, in which we refer to the unit vectors in the positive directions of the lines of curvature as the principal vectors.

Let $r_{1} \xi^{(1)}, r_{2} \xi^{(2)}, \zeta$ be three vectors whose components are functions of $u$ and $v$, and suppose that $\xi^{(1)}, \xi^{(2)}, \zeta$ are unit vectors which form a rectangular trihedral having the same disposition as that of the axes. Form the quantities

$$
\begin{aligned}
& A_{1}=-r_{2}\left(\zeta_{u} \mid \xi^{(2)}\right), \quad B_{1}=-r_{2}\left(\zeta_{v} \mid \xi^{(2)}\right), \\
& A_{2}=-r_{1}\left(\zeta_{u} \mid \xi^{(1)}\right), \quad B_{2}=-r_{1}\left(\zeta_{v} \mid \xi^{(1)}\right), \\
& D=A_{2} B_{1}-A_{1} B_{2}=r_{1} r_{2}\left(\xi^{(1)} \xi^{(2)} \widetilde{\zeta}_{u} \zeta_{v}\right),
\end{aligned}
$$


and substitute them in the expressions for $\partial / \partial s_{1}, \partial / \partial s_{2}, \nabla / \nabla s_{1}$, $\nabla / \nabla s_{2}$ given in (42). Then, if the equation

$$
\frac{\Delta \xi^{(1)}}{\Delta s_{2}}=\frac{\Delta \xi^{(2)}}{\Delta s_{1}}
$$

is satisfied, there exists a surface, uniquely determined to within a translation, whose normal vector is $\zeta$ and whose principal vectors and principal radii of normal curvature are, respectively, $\xi^{(1)}, \xi^{(2)}$, and $r_{1}, r_{2}$.

Since equation (45) is satisfied, the equations

$$
\frac{\partial x}{\partial s_{1}}=\xi^{(1)}, \quad \frac{\partial x}{\partial s_{2}}=\xi^{(2)},
$$

where $\partial / \partial s_{1}, \partial / \partial s_{2}$ are defined as in the theorem, are integrable. A surface $x=x(u, v)$ is thus determined to within a translation.

According to (46), the unit vector normal to this surface is $\zeta$. Since, by the definitions of $A_{1}, B_{1}, A_{2}, B_{2}$, given in the theorem,

or

$$
-\zeta_{u}=\frac{A_{2}}{r_{1}} \xi^{(1)}+\frac{A_{1}}{r_{2}} \xi^{(2)}, \quad-\zeta_{v}=\frac{B_{2}}{r_{1}} \xi^{(1)}+\frac{B_{1}}{r_{2}} \xi^{(2)},
$$

$$
\frac{\partial \zeta}{\partial s_{1}}=-\frac{1}{r_{1}} \xi^{(1)}, \frac{\partial \zeta}{\partial s_{2}}=-\frac{1}{r_{2}} \xi^{(2)},
$$

it follows that the surface has $\xi^{(1)}, \xi^{(2)}$ as its principal vectors and $1 / r_{1}, 1 / r_{2}$ as its principal normal curvatures.

It follows from the theorem that $r_{1} \xi^{(1)}, r_{2} \xi^{(2)}, \zeta$ form a complete system of invariant vectors, or that the vectors $\xi^{(1)}, \xi^{(2)}, \zeta$ and the functions $r_{1}, r_{2}$ form a complete system of invariants, for a given surface.

A similar theorem may be stated in the case of an arbitrary orthogonal system. Here, it is the vectors $\xi, \xi^{\prime}, \zeta$ and the functions $1 / r, 1 / r^{\prime}, 1 / \tau$ which form the complete system of invariants.

14. Integrating Factors. From $\S 8$, we have the following result.

* It is assumed that $r_{1} r_{2}(\xi^{(1)} \xi^{(2)} \overbrace{u} \zeta_{v})>0$; in particular, the case of a developable surface is excluded. 
Conditions necessary and sufficient that $m(u, v)$ and $n(u, v)$ be respectively integrating factors of $A d u+B d v$ and $A^{\prime} d u+B^{\prime} d v$ are

or

$$
\frac{\nabla m}{\nabla s}=0, \quad \frac{\nabla n}{\nabla s^{\prime}}=0,
$$

$$
\frac{\partial \log m}{\partial s}=-\frac{1}{\rho^{\prime}}, \quad \frac{\partial \log n}{\partial s^{\prime}}=\frac{1}{\rho} .
$$

A corresponding theorem is valid in the case of the canonical linear differential forms $A_{1} d u+B_{1} d v$ and $A_{2} d u+B_{2} d v$ which, when equated to zero, define the lines of curvature.

These theorems form the basis for a systematic and comprehensive theory of integrating factors for the differential equations of families of curves on the surface. They enable us, not only to discuss the cases in which the families of curves can be found by quadratures, but also to exhibit the actual integrating factors.

We content ourselves with one example, that of an isometric system. If $A d u+B d v$ and $A^{\prime} d u+B^{\prime} d v$ have a common integrating factor, $m(u, v)$, then

$$
\frac{\partial \log m}{\partial s}=-\frac{1}{\rho^{\prime}}, \quad \frac{\partial \log m}{\partial s^{\prime}}=\frac{1}{\rho},
$$

and conversely. But the condition of compatibility of these equations is

$$
\frac{\nabla}{\nabla s}\left(\frac{1}{\rho}\right)+\frac{\nabla}{\nabla s^{\prime}}\left(\frac{1}{\rho^{\prime}}\right)=0 .
$$

If this condition is fulfilled, $m(u, v)$, where

$$
\log m=\int \frac{d s^{\prime}}{\rho}-\frac{d s}{\rho^{\prime}},
$$

is a common integrating factor of $A d u+B d v$ and $A^{\prime} d u+B^{\prime} d v$, and the finite equations of the curves $C$ and $C^{\prime}$ can be found by quadratures.

Furthermore, if we set

$$
m(A d u+B d v)=d v_{1}, \quad m\left(A^{\prime} d u+B^{\prime} d v\right)=d u_{1},
$$

the linear element becomes 
where

$$
d s^{2}=\frac{1}{m^{2}}\left(d u_{1}^{2}+d v_{1}^{2}\right),
$$

$$
m=\int \frac{d v_{1}}{\rho}-\frac{d u_{1}}{\rho^{\prime}} .
$$

The condition (47) that the curves $C$ and $C^{\prime}$ form an isometric system remains unaffected when ordinary derivatives are substituted for the modified derivatives. Hence, it is essentially Bonnet's condition.*

15. Formulas of Transformation from one Orthogonal System to Another. It will suffice for our purposes to develop the formulas of transformation from the lines of curvature $C_{1}, C_{2}$ to an arbitrary orthogonal system of curves $C, C^{\prime}$.

Let the angle, at an arbitrary point $P$, from the directed curve $C_{1}$ to the directed curve $C$, measured in the positive direction, be $\alpha(u, v)$. Then the vectors $\xi, \xi^{\prime}$ may be obtained from the vectors $\xi^{(1)}, \xi^{(2)}$ by rotating these vectors about $P$ in the tangent plane through the angle $\alpha$. Thus:

$$
\begin{aligned}
& \xi=\xi^{(1)} \cos \alpha+\xi^{(2)} \sin \alpha, \\
& \xi^{\prime}=-\xi^{(1)} \sin \alpha+\xi^{(2)} \cos \alpha .
\end{aligned}
$$

Since, by (29),

$$
\begin{aligned}
& A^{\prime} \xi+A \xi^{\prime}=A_{2} \xi^{(1)}+A_{1} \xi^{(2)}, \\
& B^{\prime} \xi+B \xi^{\prime}=B_{2} \xi^{(1)}+B_{1} \xi^{(2)},
\end{aligned}
$$

we obtain, as the transformation of $A_{1} d u+B_{1} d v, A_{2} d u+B_{2} d v$ into $A d u+B d v, A^{\prime} d u+B^{\prime} d v$ :

$$
\begin{aligned}
& A d u+B d v=\left(A_{1} d u+B_{1} d v\right) \cos \alpha-\left(A_{2} d u+B_{2} d v\right) \sin \alpha \\
& A^{\prime} d u+B^{\prime} d v=\left(A_{1} d u+B_{1} d v\right) \sin \alpha+\left(A_{2} d u+B_{2} d v\right) \cos \alpha
\end{aligned}
$$

* Condition (47) becomes, when for $1 / \rho$ and $1 / \rho^{\prime}$ are substituted the value given by (16a),

or

$$
\frac{\nabla}{\nabla s} \frac{\partial}{\partial s^{\prime}} \log \left(\Delta_{1} \psi\right)^{1 / 2}-\frac{\nabla}{\nabla s^{\prime}} \frac{\partial}{\partial s} \log \left(\Delta_{1} \phi\right)^{1 / 2}=0,
$$

$$
\frac{\nabla}{\nabla s^{\prime}} \frac{\partial}{\partial s} \log \left(\frac{\Delta_{1} \psi}{\Delta_{1} \phi}\right)^{1 / 2}=0 .
$$

This is the invariant form of the usual condition to the effect that, when the linear element is $E d u^{2}+G d v^{2}$, the parametric curves form an isometric system if and only if $\log (E / G)$ is of the form $U(u)+V(v)$. 
The formulas of transformation of the ordinary directional derivatives are

$$
\begin{aligned}
& \frac{\partial \chi}{\partial s}=\frac{\partial \chi}{\partial s_{1}} \cos \alpha+\frac{\partial \chi}{\partial s_{2}} \sin \alpha \\
& \frac{\partial \chi}{\partial s^{\prime}}=-\frac{\partial \chi}{\partial s_{1}} \sin \alpha+\frac{\partial \chi}{\partial s_{2}} \cos \alpha
\end{aligned}
$$

and those of the geodesic curvatures,

$$
\begin{aligned}
& \frac{1}{\rho}-\frac{\partial \alpha}{\partial s}=\frac{\cos \alpha}{\rho_{1}}+\frac{\sin \alpha}{\rho_{2}}, \\
& \frac{1}{\rho^{\prime}}-\frac{\partial \alpha}{\partial s^{\prime}}=-\frac{\sin \alpha}{\rho_{1}}+\frac{\cos \alpha}{\rho_{2}} .
\end{aligned}
$$

These formulas are readily obtained from (30) by replacing $A, B, A^{\prime}, B^{\prime}$ by their values in terms of $A_{1}, B_{1}, A_{2}, B_{2}$, as read off from (49).

The formulas for the transformation of the modified derivatives are now readily found to be

$$
\begin{aligned}
& \frac{\nabla \chi}{\nabla s}-\chi \frac{\partial \alpha}{\partial s^{\prime}}=\frac{\nabla \chi}{\nabla s_{1}} \cos \alpha+\frac{\nabla \chi}{\nabla s_{2}} \sin \alpha, \\
& \frac{\nabla \chi}{\nabla s^{\prime}}+\chi \frac{\partial \alpha}{\partial s}=-\frac{\nabla \chi}{\nabla s_{1}} \sin \alpha+\frac{\nabla \chi}{\nabla s_{2}} \cos \alpha .
\end{aligned}
$$

It is interesting, also, to note that Euler's formulas,

$$
\frac{1}{r}=\frac{\cos ^{2} \alpha}{r_{1}}+\frac{\sin ^{2} \alpha}{r_{2}}, \quad \frac{1}{r^{\prime}}=\frac{\sin ^{2} \alpha}{r_{1}}+\frac{\cos ^{2} \alpha}{r_{2}},
$$

and the corresponding formula for the geodesic torsion, namely,

$$
\frac{1}{\tau}=\frac{1}{2}\left(\frac{1}{r_{1}}-\frac{1}{r_{2}}\right) \sin 2 \alpha,
$$

are a direct result of our theory.

For example, if we set for $\xi^{\prime}$ and $\partial \zeta / \partial s$, in

$$
\frac{1}{\tau}=\left(\xi^{\prime} \mid \frac{\partial \zeta}{\partial s}\right)
$$


their values, as given by (48) and (50), in terms of $\xi^{(1)}, \xi^{(2)}$, $\partial \zeta / \partial s_{1}, \partial \zeta / \partial s_{2}$, and replace $\partial \zeta / \partial s_{1}$ and $\partial \zeta / \partial s_{2}$ by their values from (43), we obtain immediately formula (54).*

From (53) and (54) we verify that

$$
K=\frac{1}{r r^{\prime}}-\frac{1}{\tau^{2}}
$$

From these same formulas we also find the relation

$$
\left(\frac{1}{r}-\frac{1}{r^{\prime}}\right) \tan 2 \alpha=\frac{2}{\tau},
$$

which defines the angle $\alpha$ in terms of quantities pertaining only to the curves $C$ and $C^{\prime}$. Thus, when an orthogonal system of curves $C$ and $C^{\prime}$ is given, as in $\$ 10$, by its fundamental quantities, it is possible to determine precisely the position of the system with reference to the lines of curvature.

It is evident, conversely, that the foregoing formulas permit us to pass from the fundamental quantities $A_{1}, B_{1}, A_{2}, B_{2}$, $1 / r_{1}, 1 / r_{2}$ for the lines of curvature to the fundamental quantities $A, B, A^{\prime}, B^{\prime}, 1 / r, 1 / r^{\prime}, 1 / \tau$ for any orthogonal system of curves $C$ and $C^{\prime}$, provided we know the angle $\alpha(u, v)$ under which the curves of this system meet the lines of curvature. In particular, the canonical differential equations of the curves $C$ and $C^{\prime}$ are readily found, when those of the lines of curvature are known. But the canonical differential equations of the lines of curvature are easily obtained, by algebraic processes. In fact, we have

$$
\begin{array}{rlrl}
A_{1}{ }^{2} & =\frac{1}{L}\left(\frac{E}{r_{1}}-e\right) \geqq 0, & A_{2}^{2}=\frac{1}{L}\left(e-\frac{E}{m}\right) \geqq 0, \\
A_{1} B_{1}=\frac{1}{L}\left(\frac{F}{r_{1}}-f\right), & A_{2} B_{2}=\frac{1}{L}\left(f-\frac{F}{r_{2}}\right), \\
B_{1}{ }^{2} & =\frac{1}{L}\left(\frac{G}{r_{1}}-g\right) \geqq 0, & B_{2}^{2}=\frac{1}{L}\left(g-\frac{G}{r_{2}}\right) \geqq 0,
\end{array}
$$

where

* The general method of using directional derivatives to establish formulas (53) and (54) is ascribed to Enneper; see Lilienthal, Encyclopädie der Mathematischen Wissenschaften, III D3, p. 168. 


$$
L=\frac{1}{r_{1}}-\frac{1}{r_{2}} . *
$$

Since $A_{2} B_{1}-A_{1} B_{2}=D$, we may, in extracting the square roots of $A_{1}{ }^{2}, B_{1}{ }^{2}, A_{2}{ }^{2}, B_{2}^{2}$, choose only one sign at pleasure. $\dagger$ The other three are then uniquely determined.

16. Differential Parameters. The Significance of the Modified Directional Derivatives. Let us consider, first, differential parameters with respect to the canonical linear forms $A d u+B d v$ and $A^{\prime} d u+B^{\prime} d v$, which, when equated to zero, define an orthogonal system of curves on $S$.

We single out two differential parameters of the first order with respect to these forms, namely, the derivatives of a function $\phi(u, v)$ in the positive directions of the curves $C$ and $C^{\prime}$ :

$$
\frac{\partial \phi}{\partial s}=\frac{1}{D}\left(B \phi_{u}-A \phi_{v}\right), \quad \frac{\partial \phi}{\partial s^{\prime}}=-\frac{1}{D}\left(B^{\prime} \phi_{u}-A^{\prime} \phi_{v}\right),
$$

and four differential parameters of the second order:

$$
\frac{\nabla}{\nabla s} \frac{\partial \phi}{\partial s}, \quad \frac{\nabla}{\nabla s^{\prime}} \frac{\partial \phi}{\partial s}, \quad \frac{\nabla}{\nabla s} \frac{\partial \phi}{\partial s^{\prime}}, \quad \frac{\nabla}{\nabla s^{\prime}} \frac{\partial \phi}{\partial s^{\prime}},
$$

the second and third of which are identical.

The fundamental differential parameters of the first and second order with respect to the linear element of the surface may be expressed in terms of these parameters related to the linear forms. We have

$$
\begin{aligned}
\Delta_{1} \phi & =\left(\frac{\partial \phi}{\partial s}\right)^{2}+\left(\frac{\partial \phi}{\partial s^{\prime}}\right)^{2}, \\
\Delta_{1}(\phi, \psi) & =\frac{\partial \phi}{\partial s} \frac{\partial \psi}{\partial s}+\frac{\partial \phi}{\partial s^{\prime}} \frac{\partial \psi}{\partial s^{\prime}}, \\
\Theta_{1}(\phi, \psi) & =\frac{\partial \phi}{\partial s} \frac{\partial \psi}{\partial s^{\prime}}-\frac{\partial \phi}{\partial s^{\prime}} \frac{\partial \psi}{\partial s}
\end{aligned}
$$

and

* The cases of the sphere and the plane are excluded.

$\dagger$ Geometrically: we may fix the positive direction on the lines of curvatures of one family; then that for the other family is determined. 


$$
\Delta_{2} \phi=\frac{\nabla}{\nabla s} \frac{\partial \phi}{\partial s}+\frac{\nabla}{\nabla s^{\prime}} \frac{\partial \phi}{\partial s^{\prime}} .
$$

To verify these equations, we have merely to expand the right-hand sides, and recall that

$$
E=A^{2}+A^{\prime 2}, \quad F=A B+A^{\prime} B^{\prime}, \quad G=B^{2}+B^{\prime 2} .
$$

We are now in a position to develop on $S$ the theory which is linked with the ideas of gradient, divergence, and curl, and to express it in an analytic form which is strikingly similar to that in the Cartesian plane. Let $V$ be a vector which is defined at each point of $S$, and let the components of $V$ with respect to $\xi$ and $\xi^{\prime}$ be $Q(u, v)$ and $Q^{\prime}(u, v)$,

$$
V=Q \xi+Q^{\prime} \xi^{\prime}
$$

The divergence and curl of the vector $V$ shall be defined as the expressions:

$$
\begin{aligned}
& \operatorname{div} V=\frac{\nabla Q}{\nabla s}+\frac{\nabla Q^{\prime}}{\nabla s^{\prime}}, \\
& \operatorname{curl} V=\frac{\nabla Q^{\prime}}{\nabla s}-\frac{\nabla Q}{\nabla s} .
\end{aligned}
$$

In justification of the definitions, we shall show that the expressions in question are invariant with respect to a transformation from one orthogonal system to a second. Let the components of $V$ with respect to the unit vectors $\xi^{(1)}, \xi^{(2)}$ in the directions of the lines of curvature be $Q_{1}$ and $Q_{2}$ :

$$
V=Q_{1} \xi^{(1)}+Q_{2} \xi^{(2)} \text {. }
$$

Using the formulas of transformation (48) of $\xi^{(1)}, \xi^{(2)}$ into $\xi, \xi^{\prime}$, we find

$$
Q=Q_{1} \cos \alpha+Q_{2} \sin \alpha, \quad Q^{\prime}=-Q_{1} \sin \alpha+Q_{2} \cos \alpha,
$$

and hence deduce, by means of the transformation (52) of the modified directional derivatives, the desired relations:

$$
\begin{aligned}
& \frac{\nabla Q}{\nabla s}+\frac{\nabla Q^{\prime}}{\nabla s^{\prime}}=\frac{\nabla Q_{1}}{\nabla s_{1}}+\frac{\nabla Q_{2}}{\nabla s_{2}}, \\
& \frac{\nabla Q^{\prime}}{\nabla s}-\frac{\nabla Q}{\nabla s^{\prime}}=\frac{\nabla Q_{2}}{\nabla s_{1}}-\frac{\nabla Q_{1}}{\nabla s_{2}} .
\end{aligned}
$$


The expressions (57) are the inner and outer products* of the vector operator

$$
\Delta=\xi \frac{\nabla}{\nabla s}+\xi^{\prime} \frac{\Delta}{\nabla s^{\prime}}
$$

with the vector $V$. This operator is, then, a generalization of the vector operator in the plane which is denoted by the same symbol.

Another vector operator suggests itself, namely,

$$
D=\xi \frac{\partial}{\partial s}+\xi^{\prime} \frac{\partial}{\partial s^{\prime}}
$$

and this operator, too, is of value. In fact, if $\phi(u, v)$ is a scalar function, the vector

$$
D \phi=\frac{\partial \phi}{\partial s} \xi+\frac{\partial \phi}{\partial s^{\prime}} \xi^{\prime}
$$

is the gradient of $\phi$.

The operator $D$, based on ordinary directional derivatives, applies to functions and yields vectors, - their gradients. The operator $\nabla$, based on the modified directional derivatives, applies to vectors and yields functions,-their curls and divergence.

This result sets forth clearly the significance of the modified derivatives and the relationship between them and the ordinary derivatives.

Since $D$ applies to functions and yields vectors, and $\nabla$ applies to vectors and yields functions, the two operators can be applied in turn as often as one desires.

We can, for example, apply $\nabla$ to the gradient $D \phi$ of a function $\phi$, to find its divergence and curl.

The divergence of the gradient of $\phi(u, v)$ is the Laplacian

$$
\operatorname{div} D \phi \equiv \frac{\nabla}{\nabla s} \frac{\partial \phi}{\partial s}+\frac{\nabla}{\nabla s^{\prime}} \frac{\partial \phi}{\partial s^{\prime}} .
$$

The curl of the gradient of $\phi(u, v)$ is identically zero,

* We assume hat we are dealing with two-dimensional vectors. Then the outer product, as well as the inner product, is a scalar. 


$$
\operatorname{curl} D \phi \equiv \frac{\nabla}{\nabla s} \frac{\partial \phi}{\partial s^{\prime}}-\frac{\nabla}{\nabla s^{\prime}} \frac{\partial \phi}{\partial s} \equiv 0 .
$$

Conversely, if the curl of a vector vanishes identically, the vector is the gradient of a function.

These results are, of course, direct generalizations of classical theorems in the Cartesian plane.

Since the gradients $D \phi, D \psi$ of two invariant functions are invariant vectors, their inner and outer products are invariant functions. In fact, according to (55), these products are the differential parameters of the first order of $\phi, \psi$ with respect to the linear element. On the other hand, the divergence of $D \phi$ is $\Delta_{2} \phi$.

All the differential parameters, with respect to the linear element, of a given set of functions result from the operations of inner and outer multiplication, together with the operation of finding divergence, applied to the gradients of the given functions and all functions derived from them by these operations.

The process of finding the divergence is simply that of inner multiplication by the operator $\nabla$. Hence, we have reduced the operations necessary to obtain the differential parameters with respect to the linear element to ordinary inner and outer multiplication of two-dimensional vectors, supplemented by the two types of differentiation.

17. The Geodesic Curvature Vector. The vector whose components in the directions of the curves of an orthogonal system to which the surface is referred are the geodesic curvatures of these curves shall be called the geodesic curvature vector associated with the orthogonal system. Thus,

$$
\Gamma_{C_{1} C_{2}}=\frac{1}{\rho_{1}} \xi^{(1)}+\frac{1}{\rho_{2}} \xi^{(2)}
$$

is the geodesic curvature vector associated with the lines of curvature, and

$$
\Gamma_{C_{1} C^{\prime}}=\frac{1}{\rho} \xi+\frac{1}{\rho^{\prime}} \xi^{\prime}
$$

is that associated with the general orthogonal system consisting of the curves $C, C^{\prime}$. 
These two geodesic curvature vectors are not, in general, the same. As a matter of fact, we have

$$
\Gamma_{C C^{\prime}}=\Gamma_{C_{1} C_{2}}+D \alpha,
$$

where $D \alpha$ is the gradient of the angle $\alpha$ under which the system of curves $C, C^{\prime}$ cuts the lines of curvature.

On the other hand, since the curl of the gradient of a function vanishes,

$$
\operatorname{curl} \Gamma_{C C^{\prime}}=\operatorname{curl} \Gamma_{C_{1} C_{2}} \text {. }
$$

The common value of these curls is, by (40), the negative of the total curvature of the surface.

The curl of the geodesic curvature vector is the same for every orthogonal system of curves, and is equal to the negative of the total curvature of the surface.

It follows that a surface is a developable if and only if an arbitrarily chosen geodesic curvature vector is the gradient of a function.

HARVARD UNIVERSITY 